\title{
Analysis of cosmic-ray events with ALICE at LHC
}

\author{
M. Rodríguez Cahuantzi ${ }^{\mathrm{a}}$ for the ALICE Collaboration \\ Physics Department, CINVESTAV, PO Box, 14740 México, D.F.
}

\begin{abstract}
ALICE is one of the four main experiments of the LHC at CERN. Located 40 meters underground, with $30 \mathrm{~m}$ of overburden rock, it can also operate to detect muons produced by cosmic-ray interactions in the atmosphere. An analysis of the data collected with cosmic-ray triggers from 2010 to 2013, corresponding to about 31 days of live time, is presented. Making use of the ability of the Time Projection Chamber (TPC) to track large numbers of charged particles, a special emphasis is given to the study of muon bundles, and in particular to events with high-muon density.
\end{abstract}

\section{Introduction}

The study of atmospheric muons using collider experiments started during the era of the Large Electron Positron collider (LEP) by ALEPH, DELPHI and L3 collaborations. The main result reported by the LEP experiments in the area of cosmic-ray physics was the observation of muon-bundle events that could not be described within the existing hadronic interaction models. Even with the combination of extreme assumptions of highest-measured flux value and pure-iron spectrum, the Monte Carlo models failed to describe the abundance of events with high multiplicity of muons [1].

A development of these programs is possible at the LHC, where experiments operate for many years, with the possibility of recording a large amount of cosmic-ray data. In this context, although the main purpose of ALICE is the study of beam-induced collisions, the experiment can also operate to detect muons produced by cosmic-ray interactions with the atmosphere [2]. Therefore, ALICE began a program to record cosmic-ray data, collecting about 31 effective days of data between 2010 and 2013 (around 22.5 million events recorded), during pauses in LHC operation. Additionally, in 2012 a special trigger configuration allowed a test of the detection of cosmic events during proton-proton collisions runs.

The muons collected in ALICE are created in an extensive air shower (EAS) developing after the interaction of the primary cosmic ray with the nuclei of the atmosphere. The energy of the primary cosmic ray that produces these muons is between $10^{12} \mathrm{eV}$ and $10^{18} \mathrm{eV}$. The EAS observed by ALICE with muon multiplicity larger than four, are due to primaries with energy $E>10^{14} \mathrm{eV}$.

The detection of EAS has been done by many largearea apparatus located at the surface ([3-5]), while deepunderground detectors ([6-8]) have studied the high energy muons of the EAS. The big advantages of the surface apparatus are their large size and the possibility of measuring different particles, such as electrons, muons and hadrons created in the EAS. Since the detectors involved

\footnotetext{
a e-mail: mrodriguez@fis.cinvestav.mx
}

in collider experiments are tiny compared with the large area surface arrays, the approach and the studies have to be different. In this work we have used the ALICE Time Projection Chamber (TPC) to study multi-muon events [9].

\section{Experimental setup}

ALICE is located at Point 2 of the LHC accelerator tunnel, in a cavern placed $40 \mathrm{~m}$ underground and with $30 \mathrm{~m}$ overburden of rock, at $450 \mathrm{~m}$ above the sea level. The rock absorbs all of the electromagnetic and hadronic components of an EAS, while near vertical muons with a surface energy greater than $16 \mathrm{GeV}$ can reach the detectors [10]. ALICE is a typical collider experiment, with a solenoid magnet housing the central barrel detector, and a forward muon arm, consisting of absorbers, a large dipole magnet, and fourteen planes of tracking and triggering chambers is located outside the ALICE magnet [2].

Specific triggers have been implemented to detect atmospheric muons crossing the central barrel of the ALICE apparatus (see Fig. 1). To trigger and measure cosmic-ray events, three detectors were used: ACORDE, TOF (Time of Flight) and SPD (Silicon Pixel Detector).

ACORDE consists of an array of 60 scintillator modules located on the three top octants of the ALICE magnet. Each module is composed of two superimposed plastic scintillator paddles with an effective detection area of $0.37 \mathrm{~m}^{2}$ (see Fig. 2). The trigger is given by the coincidence of the signals in $n$ different modules ( $n$-fold coincidence) in a $100 \mathrm{~ns}$ time window. For this work, the trigger of ACORDE was configured with $n=4$.

TOF is a cylindrical MRPC (Multi-gap Resistive Plate Chamber) array, completely surrounding the TPC. The trigger requires a signal in a read-out channel (a pad) in the upper part of the TOF and another in a pad in the opposite lower part.

The silicon pixel detector (SPD) is the inner part of the Internal Tracking System (ITS) of ALICE and is made of two layers located very close from the interaction point. The trigger of SPD is generated by the coincidence of two

This is an Open Access article distributed under the terms of the Creative Commons Attribution License 4.0, which permits unrestricted use, distribution, and reproduction in any medium, provided the original work is properly cited. 


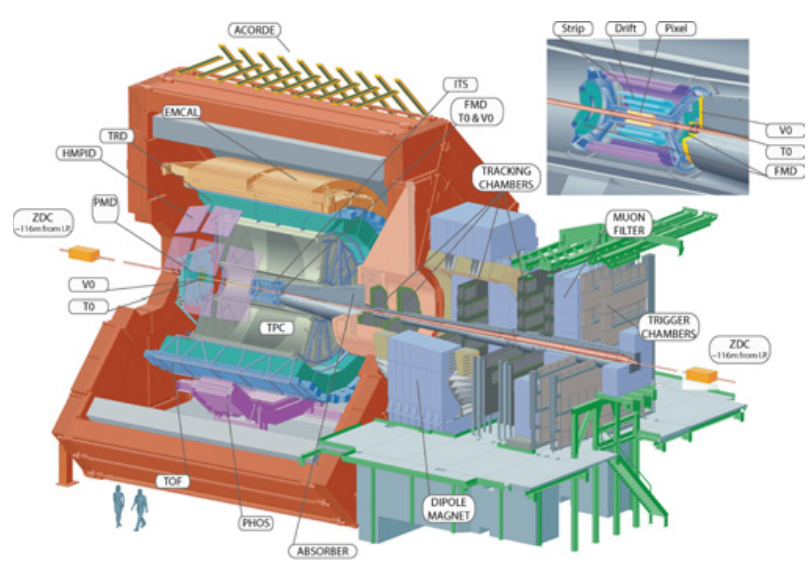

Figure 1. The ALICE detector at P2-LHC. ACORDE, TOF, TPC and SPD are used to trigger and measure cosmic-ray events.

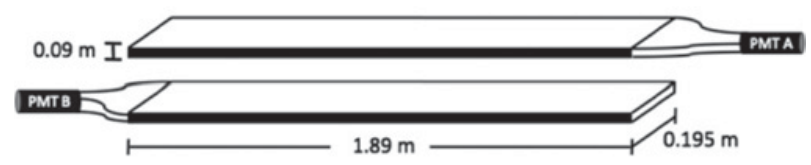

Figure 2. Schematic view of one ACORDE module. The light produced in the scintillator plastic is driven by two optical guides to one PMT per paddle. The information of the pulse is sent to the FEE (front end electronics) to generate the ACORDE cosmic-ray trigger.

signals in the top and in the bottom halves of the external layer.

A logical OR between the trigger signals of ACORDE, TOF and SPD was configured to generate the cosmic-ray trigger of ALICE.

\section{Reconstruction of atmospheric muons}

Most of the time the atmospheric muons that cross the ALICE central barrel are reconstructed as two tracks (see Fig. 3). In order to quantify the muon multiplicity per event, a dedicated algorithm was developed to match both reconstructed tracks and to obtain the full length of the muon track crossing the TPC.

There are three type of events in our data sample: single, multi-muon and interactions $\mu-F e$ (they can occur when a very energetic muon interacts with the iron of the magnet yoke of ALICE producing a shower of secondary particles crossing the TPC). For this work, only the analysis of multi-muon events is presented.

\subsection{Track reconstruction}

Each TPC track can be reconstructed with up to 159 clusters (space points). In this analysis, we required tracks with at least 50 clusters in the TPC and a momentum larger than $0.5 \mathrm{GeV} / \mathrm{c}$ in order to remove the electromagnetic background. Since the atmospheric muons coming from the same EAS event arrive almost parallel at ground level, all the analyzed tracks per event should be parallel between them.

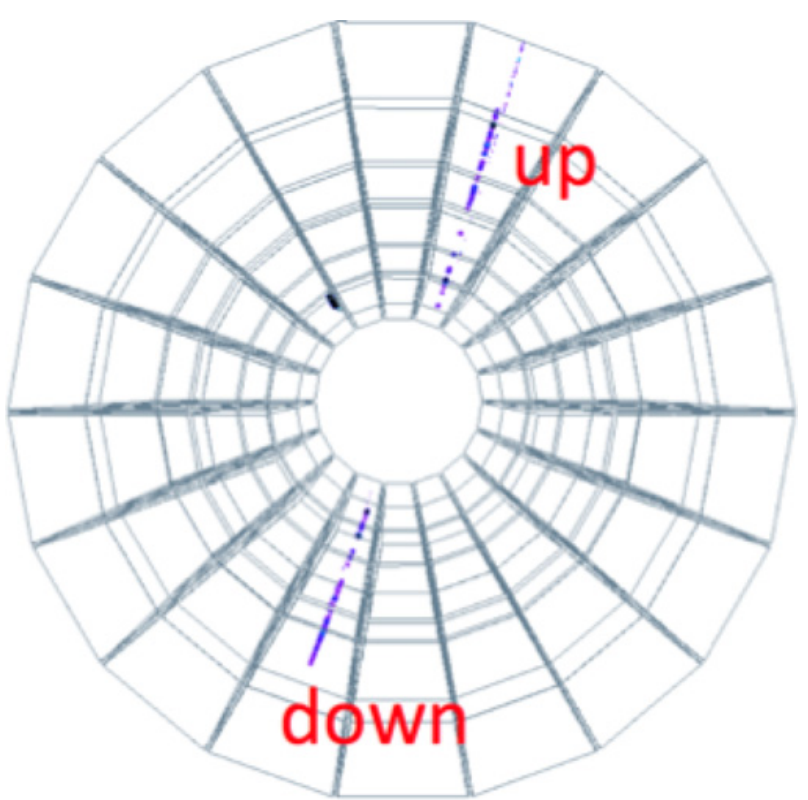

Figure 3. Track reconstruction of one single atmospheric muon with the TPC of ALICE.

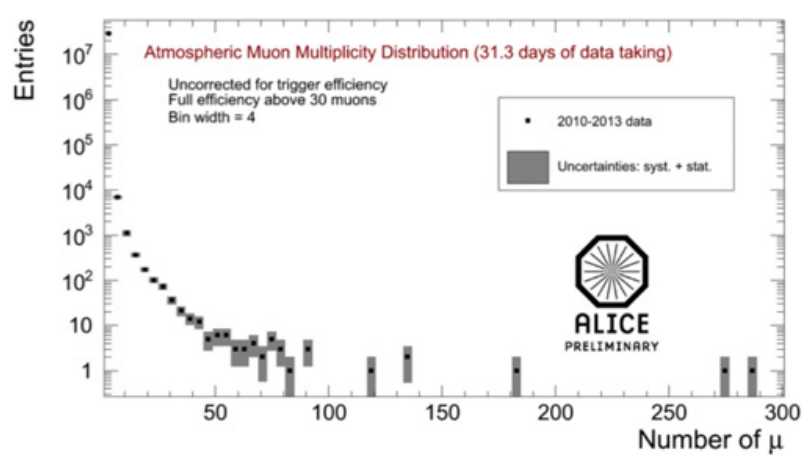

Figure 4. Atmospheric-muon multiplicity distribution (data collected by ALICE between 2010 and 2013).

Finally, each up-track is matched to the closest downtrack when the distance in the transverse plane (XZ) between them is less than $3 \mathrm{~cm}$. A muon reconstructed with two tracks is called "matched muon". If the matching condition is not fulfilled the track is anyway accepted as a muon and called "single-track muon". Most of the singletrack muons originate from particles crossing the TPC close to its edge, where part of the muon trajectory may fall outside the detector.

\section{Multiplicity distribution of atmospheric muons}

The main topic related to cosmic-ray physics investigated by ALICE is the study of the muon-multiplicity distribution and in particular of events with high density of muons.

The atmospheric-muon multiplicity (MMD) distribution from the whole sample of our data is shown in Fig. 4.

We found 6 events with a number of muons greater than 100. As expected, a smooth distribution can be 


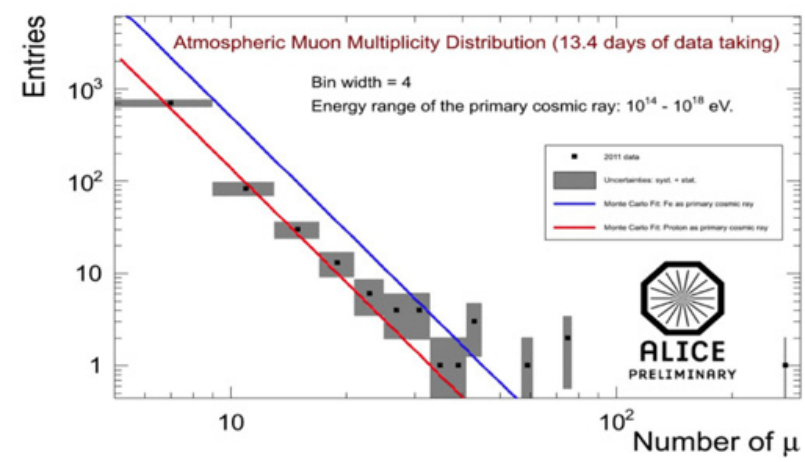

Figure 5. Atmospheric-muon multiplicity distribution of the data compared with the fit obtained with CORSIKA 6990 for proton (red line) and Fe (blue line).

observed up to a muon multiplicity around 70. The MMD reconstructed by ALICE is very similar to that obtained by the LEP experiments [1], which could not be explained by Monte Carlo models. However, it is now possible to compare our results, as will be done in the next sections, with the more sophisticated hadronic-interaction models available today.

\subsection{Monte Carlo study of MMD}

In this section, we are going to investigate the MMD, and in particular the rate and nature of high muon-multiplicity events (HME, those events with a number of muons greater than 100).

Hadronic interactions are often described through phenomenological models implemented in Monte Carlo programs. To study the MMD, we have used CORSIKA [11] version 6990 and 7350 with QGSJET as interaction model [12]. The parameters used in the Monte Carlo simulations were the following:

- two assumptions: pure p (lighter composition) and Fe (extremely heavy composition),

- usual power law spectrum $E^{-\gamma}$ with $\gamma=2.7$ for energies below the knee and $\gamma=3.0$ for energies above the knee,

- the total (all-particle) absolute flux of the cosmic rays was taken from [13],

- the core of each shower was scattered with a flat random distribution at surface level, in an area of $205 \times 205 \mathrm{~m}^{2}$ centered around the ALICE apparatus,

- the muons arriving at the surface level are propagated through the rock above ALICE taking into account the geometry at the P2-LHC,

- the muons crossing the TPC are reconstructed with the same algorithm as the real data.

We compared the MMD (only 2011 data, 13.4 days) with the Monte Carlo simulations (Corsika 6990) for an energy range of the primary cosmic ray $10^{14}<E<10^{18} \mathrm{eV}$ (the comparison with the full statistics sample shown in Fig. 4 is still in progress).

The data points are, as expected, in between the pure proton composition (light elements) and pure $\mathrm{Fe}$ (heavy elements). The lower multiplicities (lower primary energies) are closer to pure proton (see Fig. 5).
Table 1. Rate comparison of HME: Data VS Monte Carlo (QGSJET II-03).

\begin{tabular}{lll}
\hline Primary cosmic ray & HME rate [/day] & uncertainty $(\%)$ \\
\hline proton & 1 event in 11.8 days & 17 \\
Fe & 1 event in 5.7 days & 20 \\
\hline Data & 1 event in 6.3 days & 40 \\
\hline
\end{tabular}

Table 2. Rate comparison of HME: Data VS Monte Carlo (QGSJET II-04).

\begin{tabular}{lll}
\hline Primary cosmic ray & HME rate [/day] & uncertainty(\%) \\
\hline proton & 1 event in 10.7 days & 17 \\
Fe & 1 event in 4.9 days & 20 \\
\hline Data & 1 event in 6.3 days & 40 \\
\hline
\end{tabular}

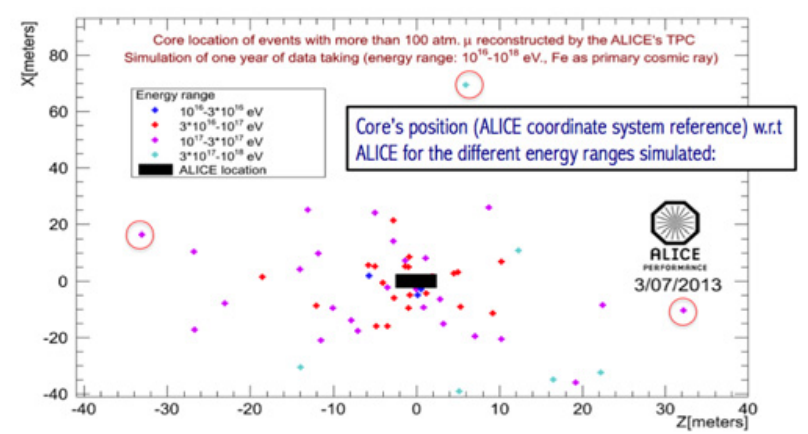

Figure 6. Core position at ALICE level of the $72 \mathrm{HME}$ of the Monte Carlo iron sample for different energy ranges and the position of ALICE (black rectangle).

To estimate the rate of the high muon-multiplicity events with the Monte Carlo models, a simulation of one year of effective data taking with Corsika 6990 (QGSJET II-03) and Corsika 7350 (QGSJET II-04, calibrated with TOTEM results on $\sigma_{p p}^{t o t / e l}$ [14]) was done:

- energy of the primary cosmic ray: $10^{16}<E<$ $10^{18} \mathrm{eV}$,

- zenith angle range $0^{\circ}-50^{\circ}$.

A preliminary estimate of the systematic uncertainties in the frequency of the events gives an error between $17 \%$ and $20 \%$. The fluctuations of these rare events are relatively small in the Monte Carlo (which corresponds to 1 year of data) but are quite large in the real data (only 31 days of data taking). Anyway, within the large uncertainties quoted in Tables 1 and 2, there is agreement between the HME rate observed in data and in the Monte Carlo calculation which makes use of CORSIKA. In Fig. 6, the position of the air shower core at ALICE level is shown for the $72 \mathrm{HME}$ of the iron Monte Carlo sample. We can notice that most of the events have the core very close to ALICE (black rectangle, less than 30 meters), few events are out of this distance (red circles).

\section{Conclusions}

In the period 2010-2013 ALICE collected around 31 days of cosmic-ray data. A mixed composition with an increasing average mass of the primary cosmic ray at higher energies is suggested by the comparison of the atmospheric muon-multiplicity distribution of data with 
the distribution obtained simulating samples of pure proton and pure iron composition.

The behavior of the MMD is in agreement with most of the experiments working in the energy range around the knee [1]. Six events of very high multiplicity have been detected in ALICE. Such high muon-multiplicity events were also found by ALEPH and DELPHI at LEP but could not be explained with the models available at that time. Using CORSIKA 6990/7350 (QGSJET II-03/04) as hadronic interaction model we are able to simulate these events and to reproduce, within relatively large uncertainties, their rate. It seems that most of the HME are due to iron or heavy nuclei with an energy greater than $10^{16} \mathrm{eV}$ and a shower core located near ALICE.

This work was supported by CONACyT posdoctoral fellow 2nd. year 2014-2015, CONACyT under grant numbers 101597, 166799 (CB 2001-1) and PAPIIT-UNAM under grant number IN108414, México. The author also thanks to the VIEP-BUAP project.

\section{References}

[1] P. Travnicek, Czech. Journal of Physics, 56 A185A200 (2006)

[2] K. Aamodt et al., (ALICE Collaboration), J. Instrum. 3, S08002 (2008)
[3] M. Aglietta et al., (EAS-TOP Collaboration), Astropart. Phys. 10 1-9 (1999)

[4] M.A.K. Glasmacher et al., (CASA-MIA Collaboration), Astropart. Phys. 10 291-302 (1999)

[5] T. Antoni et al., (KASKADE Collaboration), Astropart. Phys. 14245 (2001)

[6] G. Battistoni et. al. (NUSEX Collaboration), II Nuovo Cimento Vol. 9C, N. 2, 196-209 (1986)

[7] M. Ambrosio et. al., (MACRO Collaboration), Phys. Rev. D52 3793 (1995)

[8] P. Kuusiniemi et. al., (EMMA Collaboration), Journal of Physics: Conference Series 409012067 (2013)

[9] J. Alme et al., Nuclear Instr. and Methods in Physics Research A 622 316-367 (2010)

[10] B. Alessandro et al., (ALICE Collaboration), J. Phys, G: Nucl. Part. Phys. 321295 (2006)

[11] D. Heck et al., Report FZKA 6019 (1998), Forschungszentrum Karlsruhe, http://www-357 ik.fzk.de/corsika/physicsdescription/ corsikaphys.html

[12] S.S. Ostapchenko, Nucl. Phys. B (Proc. Suppl.) 151, 143, (2006); Phys. Rev. D 74, 014026 (2006)

[13] J. R. Horandel, Astrop. Phys. 19 193-220 (2003)

[14] G. Antchev et al. (TOTEM Collaboration), Europhys. Lett. 96, 21002 (2011) 